\title{
Molecular Composition Of Organelles In A Living Yeast Cell Studied By Confocal Raman Microspectroscopy
}

\author{
Yu-San Huang, ${ }^{*}$ Takeshi Karashima, ${ }^{* *}$ Masayuki Yamamoto** \& Hiro-o Hamaguchi* \\ *Department of Chemistry, School of Science, The University of Tokyo, 7-3-1 Hongo, Bunkyo-ku, \\ Tokyo113-033, Japan \\ **Department of Biochemistry and Biophysics, School of Science, The University of Tokyo, \\ 7-3-1 Hongo, Bunkyo-ku, Tokyo113-033, Japan
}

In order to answer the question of what molecules do really exit in organelles in a living cell, where the composition and distribution must be subject to continuous changes throughout the course of the cell cycle, a new in-vivo molecular spectroscopic method is desirable. We have used confocal Raman microspectroscopy to measure the Raman spectra directly from a single living yeast cell. Raman spectra provide detailed molecular information without the need of fluorescent tags or complicated sample preparation procedures.

The apparatus used in the present study consists of an inverted confocal microscope that is coupled with a multichannel Raman spectrometer (Tokyo Instruments, Nanofinder). The $632.8 \mathrm{~nm}$ line of a He-Ne laser is used with a $\sim 1 \mathrm{~mW}$ power at the sample point. A space resolution of $250 \mathrm{~nm}$ is achieved with a $100 \mu \mathrm{m}$ pinhole. This spatial resolution enables space-resolved Raman measurements of various organelles existing in a yeast cell. Typical measurement time is 5 min per spectrum, which is rapid enough to measure a set of Raman spectra of a cell in a particular cell period.

Space-resolved Raman spectra of a living fission yeast Schizosaccharomyces pombe in the G1/S phase of the cell cycle are shown in Figure 1. It also shows the bright-field image and fluorescence images obtained under the same microscope just before the Raman spectroscopic measurements. The bright blobs seen in the fluorescence image show the GFP-labelled nuclei and thus confirm their location. Raman spectra were measured from the seven points $A \sim G$ of the cell as shown in the bright-field image. Among the seven spectra obtained, the spectra A and B resemble with each other and so do the spectra $\mathrm{C}$ and $\mathrm{D}$, and $\mathrm{E}$ and $\mathrm{F}$. This fact indicates that the molecular composition in the yeast cell is symmetric with respect to the centre. The spectra obtained from the nuclei (A and B) are dominated by the Raman bands of proteins, while those of nucleic acid are not observed. In the spectra $C$ and $D$, strong Raman bands ascribable to phospholipuids appear at $1655 \mathrm{~cm}^{-1}, 1439 \mathrm{~cm}^{-1}$, $1300 \mathrm{~cm}^{-1}$, and $726 \mathrm{~cm}^{-1}$. The origin of the strong band observed at $1602 \mathrm{~cm}^{-1}$ is unclear. The spectrum obtained from the septum $(\mathrm{G})$ is dominated by the bands of carbohydrates.

In Figure 2, the Raman spectrum $\mathrm{C}$ is compared with that of phosphatidylcholine, the major component of the phospholipid bilayer membrane. The principal features in the spectrum $\mathrm{C}$, except for the band at $1602 \mathrm{~cm}^{-1}$, exist in the phosphatidylcholine spectrum. Considering the fact that the mitochondria are double-membrane bounded organelles, we suspect that mitochondria exist in the vicinity of the nucleus, and that they give rise to both strong phospholipid Raman bands and the unidentified Raman band at $1602 \mathrm{~cm}^{-1}$. To the best of our knowledge, there is no biological molecule that exhibits an isolated strong Raman band at $1602 \mathrm{~cm}^{-1}$. It seems that the $1602 \mathrm{~cm}^{-1}$ band arises from an unknown molecular species existing in mitochondria in a living cell. 


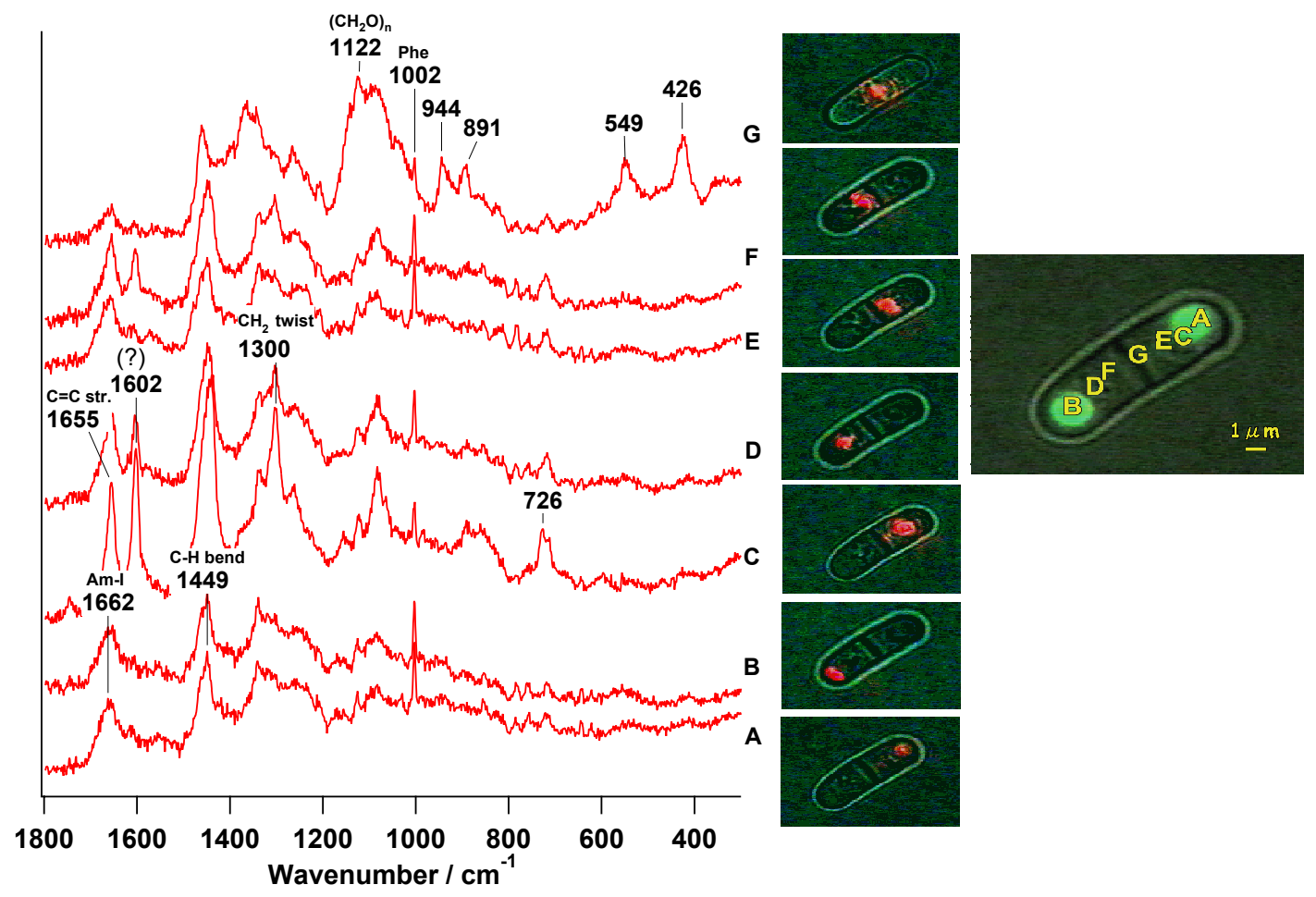

FIG. 1. The space-resolved Raman spectra of a single living yeast cell. A part of assignments of the observed Raman bands are noted in the figure.

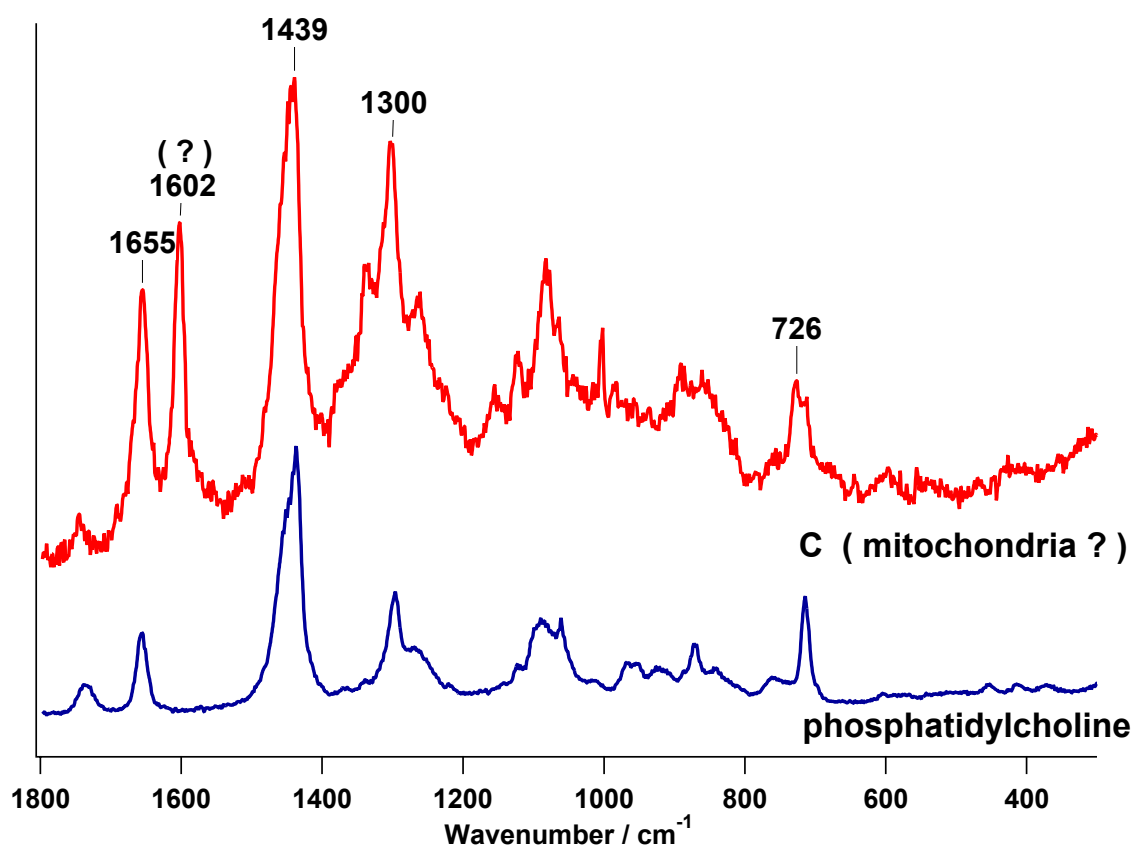

FIG. 2. Comparison of the spectrum obtained from $\mathrm{C}$ (red line) and phosphatidylcholine (blue line). We suspect that spectrum $\mathrm{C}$ originates from mitochondria existing in the vicinity of the nucleus. 